\title{
4-Dimensional Computed Tomography
}

National Cancer Institute

\section{Source}

National Cancer Institute. 4-Dimensional Computed Tomography. NCI Thesaurus. Code C116441.

Computed tomography scans designed to minimize motion artifacts. 4DCT can be accomplished by collecting data for the duration of the patient's respiratory cycle and by oversampling $\mathrm{CT}$ data acquisition at each slice. 